\title{
BEFORE THE BAR: KINEMATIC DETECTION OF A SPHEROIDAL METAL-POOR BULGE COMPONENT
}

\author{
Andrea Kunder ${ }^{1}$, R. M. Rich ${ }^{2}$, A. $\mathrm{KoCH}^{3}$, J. Storm ${ }^{1}$, D. M. NAtaF ${ }^{4}$, R. De Propris ${ }^{5}$, A. R. Walker ${ }^{6}$, G. Bono ${ }^{7,8}$, \\ C. I. JohnSON ${ }^{9}$, Juntai SHEN ${ }^{10}$, AND Z.-Y. Li ${ }^{10}$ \\ ${ }^{1}$ Leibniz-Institut für Astrophysik Potsdam (AIP), An der Sternwarte 16, D-14482 Potsdam, Germany \\ ${ }^{2}$ Department of Physics and Astronomy, University of California, Los Angeles, Los Angeles, CA 90095-1562, USA \\ ${ }^{3}$ Physics Department, Lancaster University, Lancaster LA1 4YB, UK \\ ${ }^{4}$ Research School of Astronomy and Astrophysics, The Australian National University, Canberra, ACT 2611, Australia \\ ${ }^{5}$ Finnish Centre for Astronomy with ESO, University of Turku, 2150 Turku, Finland \\ ${ }^{6}$ Cerro Tololo Inter-American Observatory, National Optical Astronomy Observatory, Casilla 603, La Serena, Chile \\ ${ }^{7}$ Dipartimento di Fisica, Universita di Roma Tor Vergata, via Della Ricerca Scientifica 1, I-00133 Roma, Italy \\ ${ }^{8}$ INAF, Rome Astronomical Observatory, via Frascati 33, I-00040, Monte Porzio Catone, Italy \\ ${ }^{9}$ Harvard-Smithsonian Center for Astrophysics, Cambridge, MA 02138, USA \\ ${ }^{10}$ Key Laboratory for Research in Galaxies and Cosmology, Shanghai Astronomical Observatory, \\ Chinese Academy of Sciences, 80 Nandan Road, Shanghai 200030, China \\ Received 2016 February 3; accepted 2016 March 18; published 2016 April 18
}

\begin{abstract}
We present 947 radial velocities of RR Lyrae variable stars in four fields located toward the Galactic bulge, observed within the data from the ongoing Bulge RR Lyrae Radial Velocity Assay (BRAVA-RR). We show that these RR Lyrae stars (RRLs) exhibit hot kinematics and null or negligible rotation and are therefore members of a separate population from the bar/pseudobulge that currently dominates the mass and luminosity of the inner Galaxy. Our RRLs predate these structures and have metallicities, kinematics, and spatial distribution that are consistent with a "classical" bulge, although we cannot yet completely rule out the possibility that they are the metal-poor tail of a more metal-rich $([\mathrm{Fe} / \mathrm{H}] \sim-1 \mathrm{dex})$ halo-bulge population. The complete catalog of radial velocities for the BRAVA-RR stars is also published electronically.
\end{abstract}

Key words: Galaxy: bulge - Galaxy: evolution - Galaxy: formation - Galaxy: halo - Galaxy: kinematics and dynamics - Galaxy: structure

Supporting material: machine-readable table

\section{INTRODUCTION}

The majority of massive galaxies $\left(>10^{9} M_{\odot}\right)$, similar to the Milky Way, have a distinct rise in surface brightness above the disk, referred to as a bulge (Fisher \& Drory 2011). Galaxy bulges are observed to either rotate rapidly like a disk and are generally referred to as pseudobulges, or they are dominated by random motions and are therefore pressure supported by a central velocity dispersion. This latter type is referred to as a classical bulge (e.g., Kormendy \& Illingworth 1982). That not all bulges are alike suggests that the bulge type of a galaxy carries significance for the galaxy's evolutionary history, such as its merger history and star formation efficiency (e.g., Martig et al. 2012; Obreja et al. 2013; Fiacconi et al. 2015). The properties of the bulge in our Galaxy are, therefore, a fundamental parameter with which to understand the formation of the Milky Way.

The first wide-area spectroscopic surveys of the Milky Way bulge have shown that it consists of a massive bar rotating as a solid body (Rich et al. 2007; Kunder et al. 2012; Ness et al. 2013; Zoccali et al. 2014). The internal kinematics of these stars are consistent with at least $90 \%$ of the inner Galaxy being part of a pseudobulge and lacking a pressure-supported, classical-like bulge (Shen et al. 2010; Ness et al. 2013). Recent studies have indicated a bimodal nature of bulges-that two bulge populations, likened to classical and pseudobulges, can exist within a galaxy, with differences being in the relative proportions of the two (Obreja et al. 2013; Fisher \& Drory 2016). There has accordingly been considerable debate about whether there is room for a classical component in the bulge (Zoccali et al. 2008; Babusiaux et al. 2010).
The oldest and most metal-poor stars (which may trace the dark matter) are thought to be found in the center of the Galaxy -in the bulge but not sharing its kinematics and abundance patterns (Tumlinson 2010). Therefore, perhaps the greatest possibility of uncovering a classical component would be within the metal-poor bulge stars. Unfortunately, spectroscopic surveys studying thousands of giants and red clump stars in the bulge have found that metal-poor stars in the bulge are rare, greatly limiting the use of metal-poor stars to uncover and probe a possible classical bulge component (Ness et al. 2013; Casey \& Schlaufmann 2015; Howes et al. 2015; Koch et al. 2016). Perhaps the easiest identifiable old, metal-poor bulge population are those horizontal branch stars that pulsate as RR Lyrae stars (RRLs). Since the absolute brightness of RRLs are known to within $\sim 10 \%$, the discovery of a significant population of RRLs toward the bulge permitted the first distance determination to the Galactic center from a stellar population (Baade 1946).

In this Letter, we report on the kinematics of a large sample of these stars in the Galactic bulge field. Our sample consists of RRLs with Galactocentric distances within $\sim 10 \%$ of the distance of the Sun to the galactic center, so our sample represents the typical "bulge" RRL population.

\section{OBSERVATIONS AND RADIAL VELOCITY}

Observations were performed using the AAOmega multifiber spectrograph on the Anglo-Australian Telescope (AAT) on 2013 May, 2013 June, 2014 June, and 2015 August, in dual beam mode centered on $8600 \AA$, with the $580 \mathrm{~V}$ and $1700 \mathrm{D}$ gratings to probe the Calcium Triplet (NOAO PropID: 2014A- 

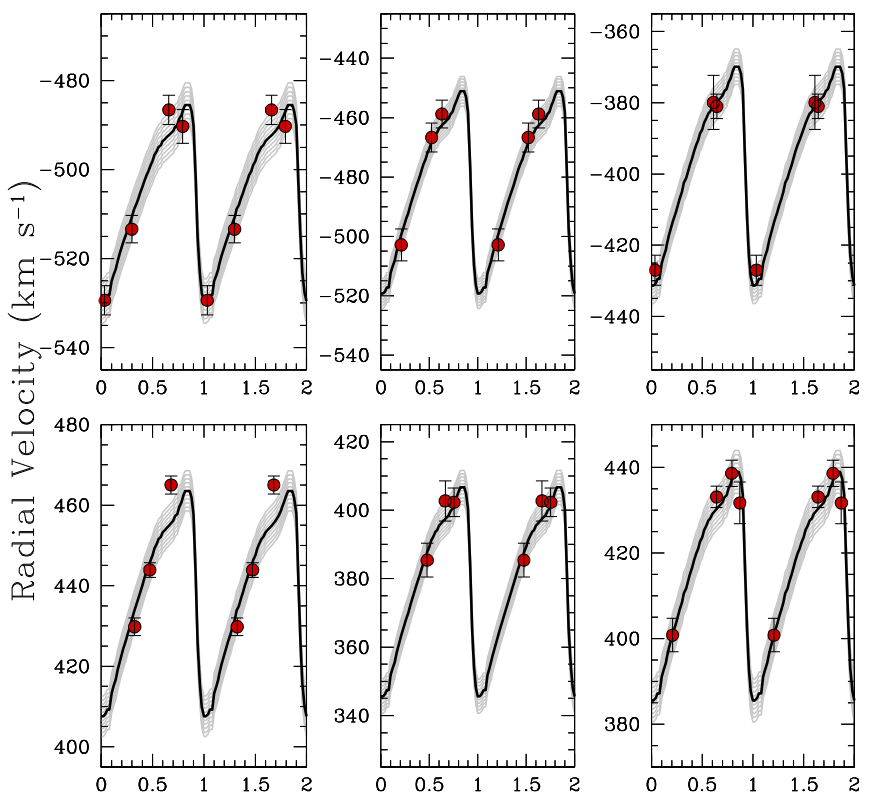

Phase

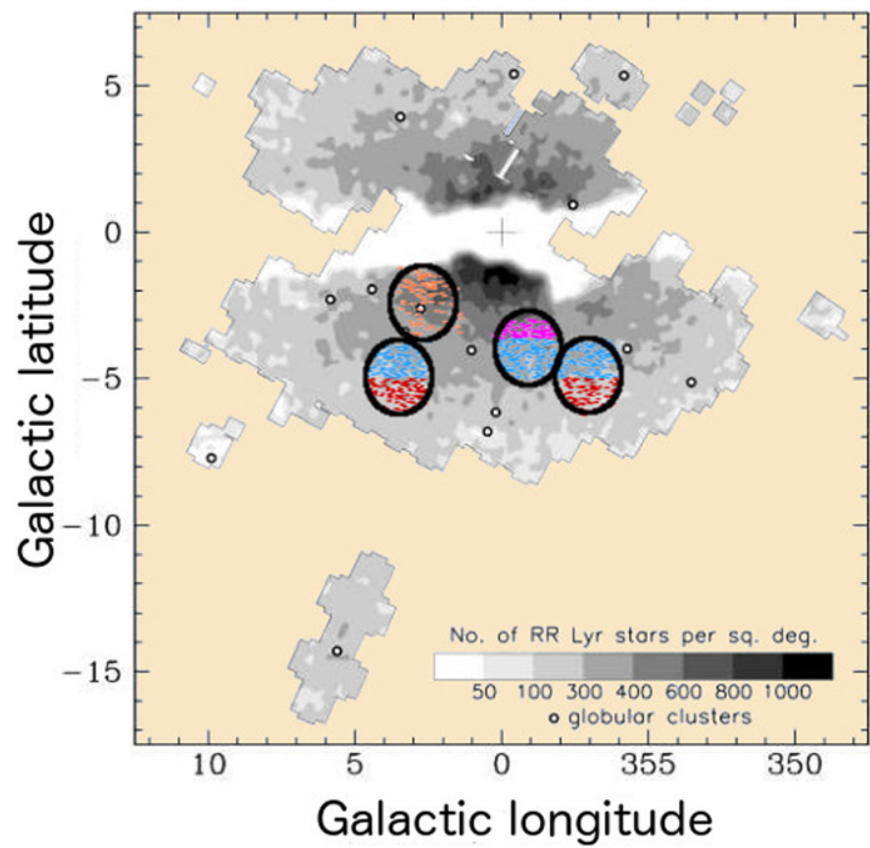

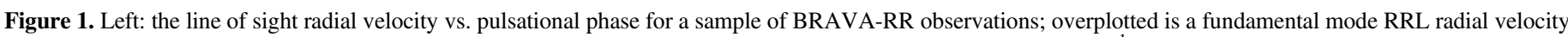

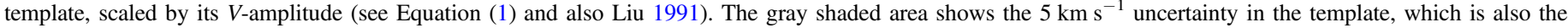

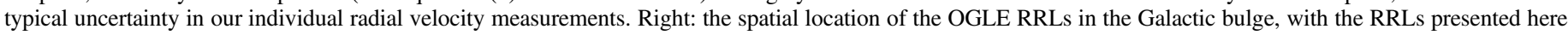

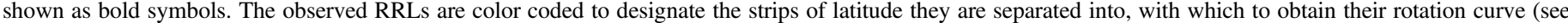
Figure 2).

0143; PI: A. Kunder and NOAO PropID: 2015B-071; PI: A. Kunder). This covers the wavelength regime of about $8300-8800 \AA$ at a resolution of $R \sim 10,000$. Exposure times were between one to two hours, and in general, there are between 2 and 5 epochs for each RRL. The 2013 observations were carried out in conjunction with a bulge survey designed for detached red giant eclipsing binary twins (AAT: 2013A-05; PI: D. Nataf). Extra fibers were available and resourcefully allocated to 95 bulge RRLs, and these RRLs have up to 15 epochs of observations.

The OGLE-III catalog of RRLs (Pietrukowicz et al. 2012) was used to select the targets. We observed all bulge fundamental mode RRLs (RR0 Lyrae stars) that were free of a companion within a 2 arcsec radius in our four fields. These have been phased by the stars known period and overplotted with the radial velocity template from Liu (1991; Figure 1). This template is scaled using a correlation between the amplitudes of velocity curves and light curve:

$$
A_{\mathrm{rv}}=\frac{40.5 \times V_{\mathrm{amp}}+42.7}{1.37}
$$

as shown in Liu (1991). Because the $I$-amplitude of the OGLE stars is known much more precisely than the $V$-amplitude, we use the relation $V_{\mathrm{amp}}=I_{\mathrm{amp}} \times 1.6$ to find the $V$-amplitude of each star (see, e.g., Table 3 in Kunder et al. 2013). The socalled "projection factor" $p=1.37$ is necessary because Liu (1991) uses pulsation velocities, which are related to observed radial velocities as $v_{\mathrm{obs}}=v_{\text {puls }} / p$. The projection factor $p$ can range from 1.31 to 1.37 (Liu 1991; Kovács 2003), and we adopted $p=1.37$ as in Sesar (2012).

Due to our sampling techniques, almost all of our stars have at least two spectra at different phases, which facilitates the fitting of a radial velocity curve to the measurements. The zero- point in phase is fixed using the time of maximum brightness as reported by OGLE-IV (Soszy'nski et al. 2014), and the pulsation curve is shifted in radial velocity until it matches the observations. More weight is given to points that fall between $\phi=0.0$ and 0.6 , as this is where the uncertainty in the radial velocity shape of the template is minimized (see, e.g., Figure 1 in Sesar 2012). More weight is also given to the observations with a higher signal to noise, which also generally occurs between $\phi=0.0$ and 0.6. The star's time-averaged velocity is determined by finding the velocity at $\phi_{\text {obs }}=0.38$ (Liu 1991).

We use 24 RRLs with well-derived radial velocities to investigate how 2-3 epochs per light curve affect our center-ofmass radial velocities. The 24 RRLs are listed in Table 1 and span a wide range of metallicities, pulsation periods, and amplitudes. Because these 24 RRLs have individual radial velocity uncertainties that are $\sim 1 \mathrm{~km} \mathrm{~s}^{-1}$, we first assign each radial velocity measurement a Gaussian uncertainty distribution of $4 \mathrm{~km} \mathrm{~s}^{-1}$ to simulate the typical errors from the observed Bulge RR Lyrae Radial Velocity Assay (BRAVA-RR) observations. We then use the Liu (1991) template and decrease the number of epochs from $\sim 100$ to 2 and measure how the center-of-mass radial velocity changes. In general, the center-of-mass radial velocity changes by $\sim 2 \mathrm{~km} \mathrm{~s}^{-1}$ or less; only in the unusual cases where there were no epochs between $\phi=0.0$ and 0.6 , but instead the observations fell on the rising branch at phases greater than 0.85 , did the center-of-mass radial velocity change by $\sim 5 \mathrm{~km} \mathrm{~s}^{-1}$.

Similar results have been shown previously; for example, Jeffery et al. (2007) showed that center-of-mass radial velocities have a typical uncertainty of $\pm 1.5 \mathrm{~km} \mathrm{~s}^{-1}$ for variables observed at least three times when using the Liu (1991) template. A visual inspection of the radial velocity curves of the 63 BRAVA-RR stars with 10 or more epochs of 
Table 1

Radial Velocity Light Curves of Local RR Lyrae Stars

\begin{tabular}{|c|c|c|c|}
\hline Name & $\begin{array}{l}\log \text { Per- } \\
\text { iod (days) }\end{array}$ & {$[\mathrm{Fe} / \mathrm{H}]$} & Source \\
\hline WY Ant & -0.240838 & -1.25 & Skillen et al. (1993) \\
\hline$X$ Ari & -0.186314 & -2.20 & Jones et al. (1987) \\
\hline RR Cet & -0.257245 & -1.25 & Liu \& Janes (1989) \\
\hline UU Cet & -0.217469 & -1.00 & Clementini et al. (1990) \\
\hline W Crt & -0.383976 & -0.70 & Skillen et al. (1993) \\
\hline DX Del & -0.325491 & -0.20 & Meylan et al. (1986) \\
\hline RX Eri & -0.231180 & -1.40 & Liu \& Janes (1989) \\
\hline SS Leo & -0.203186 & -1.51 & Fernley et al. (1990) \\
\hline RV Oct & -0.243239 & -1.75 & Skillen et al. (1993) \\
\hline V445 Oph & -0.401187 & -0.39 & Fernley et al. (1990) \\
\hline AV Peg & -0.408518 & 0.00 & Liu \& Janes (1989) \\
\hline RV Phe & -0.224454 & -1.50 & Cacciari et al. $(1987,1989)$ \\
\hline BB Pup & -0.318267 & -0.60 & Skillen et al. (1993) \\
\hline VY Ser & -0.146245 & -1.80 & Carney \& Latham (1984) \\
\hline W Tuc & -0.192305 & -1.35 & Clementini et al. (1990) \\
\hline UU Vir & -0.322752 & -0.55 & $\begin{array}{l}\text { Jones et al. (1988), Liu \& } \\
\text { Janes (1989) }\end{array}$ \\
\hline 47Tuc-V9 & -0.132620 & -0.71 & Storm et al. (1994) \\
\hline M4-V2 & -0.271092 & -1.30 & Liu \& Janes (1990) \\
\hline M4-V32 & -0.237240 & -1.30 & Liu \& Janes (1990) \\
\hline M4-V33 & -0.211245 & -1.30 & Liu \& Janes (1990) \\
\hline M5-V8 & -0.262617 & -1.40 & Storm et al. (1992) \\
\hline M5-V28 & -0.264455 & -1.40 & Storm et al. (1992) \\
\hline M92-V1 & -0.153171 & -2.24 & Storm et al. (1992) \\
\hline M92-V3 & -0.195575 & -2.24 & Storm et al. (1992) \\
\hline
\end{tabular}

observations also indicates that the Liu (1991) template is sufficient to within $5-10 \mathrm{~km}^{-1}$ to obtain a center-of-mass radial velocity. Indeed, it is impressive how well the radial velocity template fits to the diverse RRLs listed in Table 1 . We note that $87 \%$ of our BRAVA-RR stars have 3 or more epochs of observations, making it statistically unlikely that the BRAVARR stars do not have observations in the regime where the template most accurately aligns with the observed radial velocity measurements. Therefore, our center-of-mass radial velocity uncertainties are $5-10 \mathrm{~km} \mathrm{~s}^{-1}$.

Figure 1 shows example pulsation curves for the RRLs-in particular, we show those with the most extreme radial velocities to illustrate this is a kinematically hot population. Table 2 gives the OGLE ID (1), the R.A. (2), and decl. (3) as provided by OGLE, the star's time-average velocity (4), the number of epochs used for the star's time-average velocity (5), the period of the star (6), the $V$-band magnitude (7), the $I$-band magnitude (8) and the $I$-band amplitude (9) as calculated by OGLE, and last the photometric metallicity from the OGLE Iband light curve (10).

\section{DISCUSSION}

\subsection{Rotation Curve}

From spectroscopic observations of 947 RRLs in four 3 sq. deg. fields located toward the bulge, we plot the mean radial velocity and velocity dispersion for RRLs as a function of position (galactic latitude and longitude) in Figure 2. These are found to be radically different from the trends traced by the more metal-rich red giants in the BRAVA and GIBS surveys (Kunder et al. 2012; Zoccali et al. 2014) as the RRLs show null rotation and hot (high velocity dispersion) kinematics. In the $A R G O S$ survey, one observes a slowly rotating metal-poor population (Ness et al. 2013), which is hypothesized to arise from stellar contamination from disk and halo stars, as it is only seen at high galactic latitude. In contrast, our stars are at low galactic latitudes and their more certain distance estimates indicate they are within $1 \mathrm{kpc}$ of the Galactic center, where the surface-density of bulge stars is usually dominant compared to the disk and halo. We conclude that we are tracing an older, more spheroidal component in the inner Galaxy that may be likened to a classical bulge.

\subsection{Metallicity Distribution}

The $[\mathrm{Fe} / \mathrm{H}]$ metallicity distribution in our sample spans three orders of magnitude, with spectroscopic metallicities derived from the calcium triplet $8498 \AA$ line (Wallerstein et al. 2012) ranging from -2.5 to super-solar metallicities, peaking at $[\mathrm{Fe} / \mathrm{H}] \sim-1 \mathrm{dex}$. Therefore, as shown previously (Walker \& Terndrup 1991), the bulge field RRLs are on average $\sim 1$ dex more metal-poor than "normal" bar stars, yet some of the bulge field RRLs have metallicities that overlap in abundance with the bar population. The bulge field RRLs are also more metal rich than the stellar halo (e.g., An et al. 2013), although the metallicity gradient observed in the field RRLs is consistent with an inner bulge-halo at distances closer to the Galactic center being more metal rich (Suntzeff et al. 1991).

None of the bulge field RRLs are extremely metal poor, in contrast with what is predicted from a very old inner halo (Tumlinson 2010; Howes et al. 2015) there is presently no indication for many stars in our sample with $[\mathrm{Fe} / \mathrm{H}]<-3$, although the evolutionary tracks for such metal-poor stars may make it less likely for them to traverse through the instability strip, thus becoming an RR Lyrae star (e.g., Lee et al. 1994, see their Figure 1). The large metallicity spread is suggestive of multiple populations within the bulge field RRL sample (Lee et al. 2015). In Figure 2, it is clear that the metal-rich RRLs have a smaller dispersion compared to the more metal-poor stars, which indicates there were likely various RRL formation mechanisms in the bulge. This might plausibly be related to the two distinct bulge field RRL sequences in the period-amplitude diagram (Pietrukowicz et al. 2015) as well as the suggestion that only in the most central part (inner $1 \mathrm{kpc}$ ) of the bulge do the RRLs exhibit a weak bar-like substructure (Pietrukowicz et al. 2012; Dékány et al. 2013).

We note that the BRAVA-RR spectroscopic metallicities are still being finalized, and this will be the topic of a subsequent BRAVA-RR paper. Therefore throughout this Letter we use photometric metallicities obtained from a linear metallicity relationship in the pulsational period and phase difference between the first and third harmonic $\phi_{31}$ (where $\left.\phi_{n m}=m \phi_{n}-n \phi_{m}\right)$ in a Fourier decomposition of the OGLE I-band light curves (Smolec 2005). These photometric metallicites are placed on the Carretta et al. (2009) metallicity scale. The plots, however, do not change significantly when using our preliminary CaT abundances.

\subsection{Mass Estimate}

We can estimate the mass of the "old" inner Galaxy component by comparing the relative numbers of red clump giants (metal-rich horizontal branch stars) and RRLs in the OGLE-III survey (Pietrukowicz et al. 2012; Nataf et al. 2013). Here, we assume that all the red clump stars are part of the rotating bar, whereas all the RRLs are part of a non-rotating 
Table 2

Radial Velocities of BRAVA-RR Stars

\begin{tabular}{|c|c|c|c|c|c|c|c|c|c|}
\hline OGLE ID & R.A. (J2000.0) & Decl. (J2000.0) & $\mathrm{HRV}_{\phi=0.38}\left(\mathrm{~km} \mathrm{~s}^{-1}\right)$ & \# Epochs & Period (days) & $(V)_{\mathrm{mag}}$ & $(I)_{\mathrm{mag}}$ & $I_{\mathrm{amp}}$ & {$[\mathrm{Fe} / \mathrm{H}]_{\text {phot }}$} \\
\hline 06138 & 175323.86 & -341048.5 & -142 & 3 & 0.51685809 & 16.929 & 15.763 & 0.49 & -0.59 \\
\hline 06166 & 175326.77 & -340858.1 & -3 & 3 & 0.53398387 & 16.986 & 15.637 & 0.62 & -1.00 \\
\hline 06197 & 175328.89 & -341809.3 & 27 & 4 & 0.48368631 & 16.791 & 15.696 & 0.70 & -1.08 \\
\hline 06227 & 175330.50 & -342457.0 & -12 & 6 & 0.50614560 & 16.841 & 15.760 & 0.59 & -1.15 \\
\hline 06257 & 175333.61 & -335518.0 & 130 & 3 & 0.55305696 & 17.951 & 16.444 & 0.56 & -0.91 \\
\hline 06382 & 175343.89 & -341224.0 & -219 & 4 & 0.68896691 & 16.840 & 15.674 & 0.63 & -1.32 \\
\hline
\end{tabular}

(This table is available in its entirety in machine-readable form.)
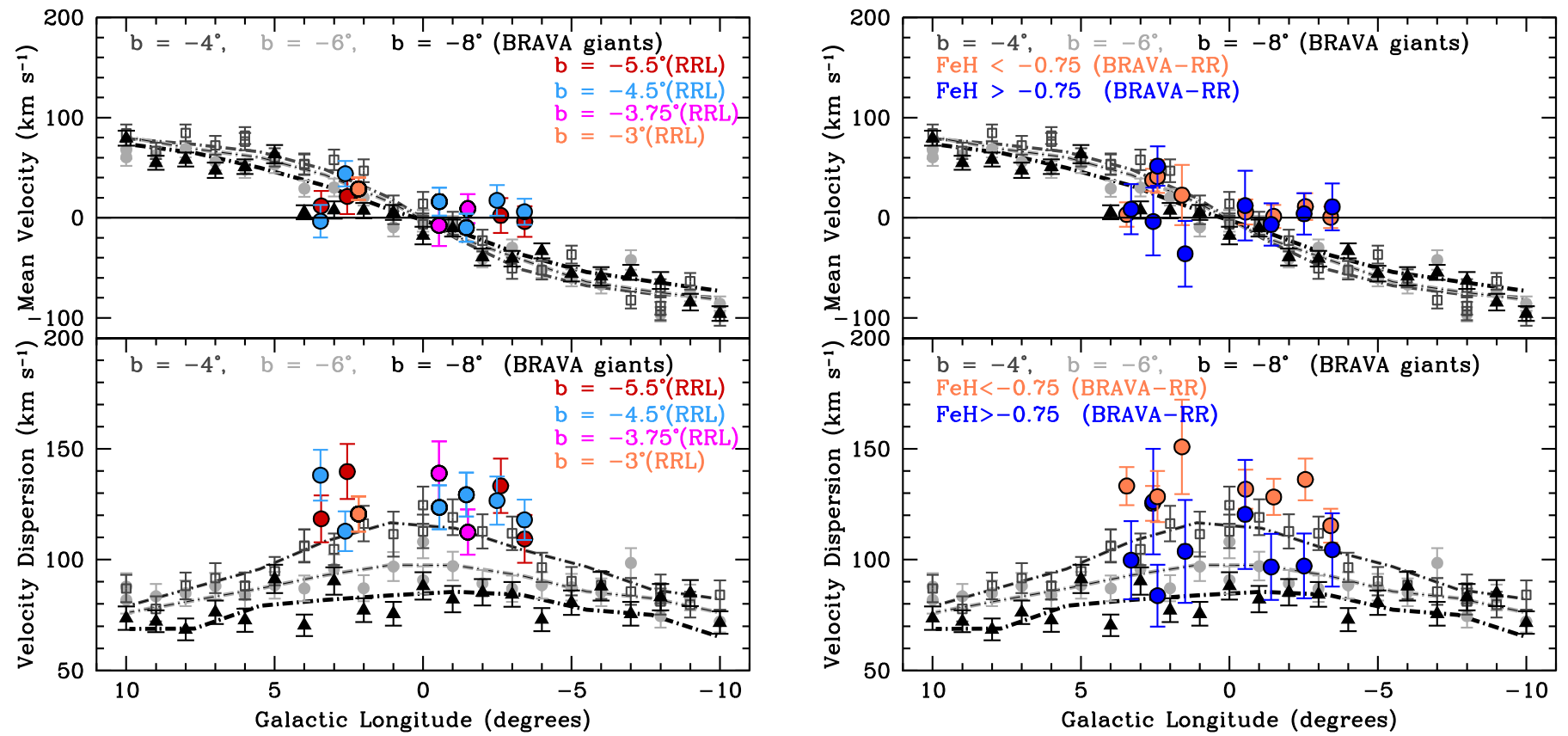

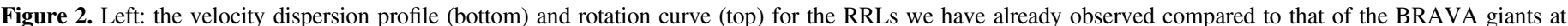

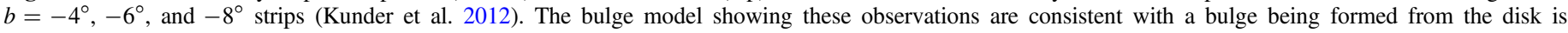

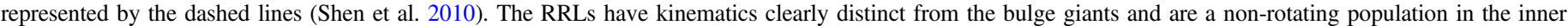

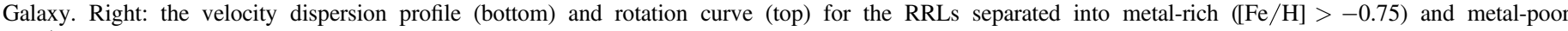
$([\mathrm{Fe} / \mathrm{H}]<-0.75)$ stars. The more metal-rich RRLs have metallicities that overlap with the pseudobulge red giants, yet they still show no substantial rotation.

component (e.g., a classical bulge). We also presume that RRLs have the same lifetime as red clump stars and were formed from stellar populations with the same IMF as the red clump stars. The kinematically hot component we recover in this Letter then amounts to $\sim 1 \%$ of the total central mass. A similar mass is calculated from the fuel consumption theorem (Renzini \& Buzzoni 1986), assuming again all the RRLs are part of a nonrotating component and have a narrow range in age. This is broadly consistent with current bulge formation models, which predict that no more than $\sim 5 \%$ of a merger-generated bulge, which is slowly rotating and dispersion supported, may exist within the Milky Way bulge (Shen et al. 2010; Ness et al. 2013; Di Matteo et al. 2015). Although such a small mass may pose a challenge in understanding how this central component could remain stationary in the much more massive bar potential (Saha \& Gerhard 2013), some dynamical studies do suggest that a hot population is only weakly affected, if at all, by the bar dynamics (Minchev et al. 2012).

\subsection{Interpretation}

Our velocities rule out that possibility that the majority of RRLs in the direction of the bulge are part of the bar. Given the ages of the RRLs (Walker 1989; Lee 1992), this indicates that the inner Galaxy component traced out by the BRAVA-RR stars is at least $\sim 1$ Gyr older than the dominant bar population.

It may be that the RRL stars toward the bulge are actually an inner halo-bulge sample, as originally speculated in the early 1990s (e.g., Minniti 1994) and as at least one RRL orbit toward the Galactic bulge seems to indicate (Kunder et al. 2015). However, the velocity dispersion of the bulge RRLs is $\sim 10 \mathrm{~km} \mathrm{~s}^{-1}$ larger than that seen in both the local RRL halo sample (Layden 1994; Beers et al. 2000) and from other halo star samples (e.g., Battaglia et al. 2006; Brown et al. 2009). In fact, we are not aware of any other stellar population in the galaxy with a larger velocity dispersion than that of the BRAVA-RR stars. The decrease in velocity dispersion with metallicity (seen in Figures 2 and 3) is also characteristic of 

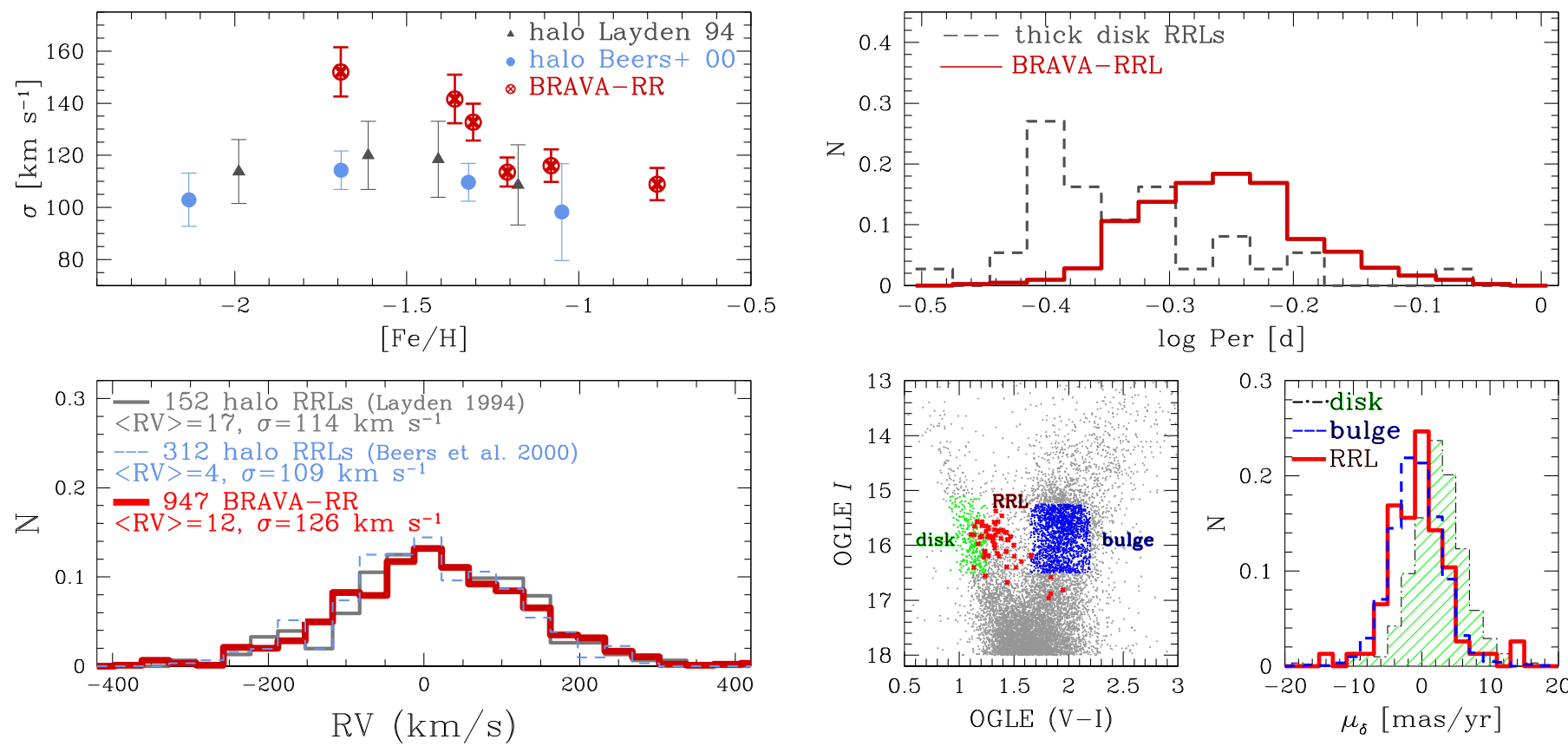

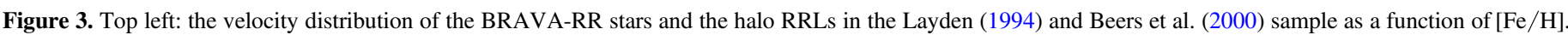

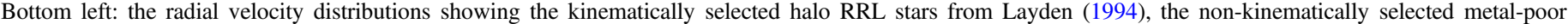

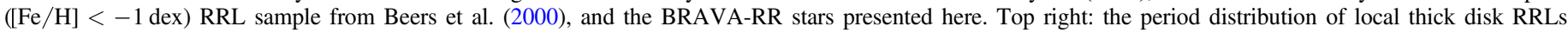

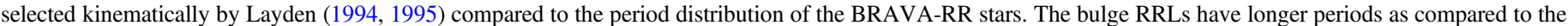

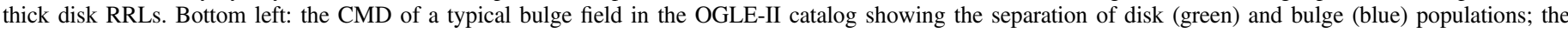

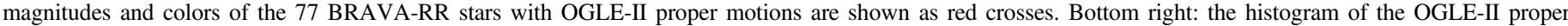

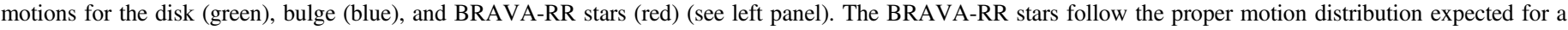
typical bulge population.

stars located in the bulge regions (e.g., Rich 1990; Babusiaux et al. 2010; Johnson et al. 2011). In contrast, the velocity dispersion of halo stars either does not change (e.g., Noris 1986) or does not change as significantly (e.g., Chiba \& Beers 2000; Kafle et al. 2013) as a function of metal abundance. A comparison of velocity dispersion with $[\mathrm{Fe} / \mathrm{H}]$ for the BRAVA-RR stars and for the halo RRL star sample of Layden (1994) and Beers et al. (2000) is shown explicitly in Figure 3 (left panel). If the RRL stars toward the bulge are an inner halo-bulge population, this component would be the most metal-rich halo population identified in the Galaxy, with a mean $[\mathrm{Fe} / \mathrm{H}] \sim-1 \mathrm{dex}$, compared to the inner halo $([\mathrm{Fe} / \mathrm{H}] \sim-1.6 \mathrm{dex})$ and the outer halo $([\mathrm{Fe} / \mathrm{H}] \sim-2.2$ dex) (e.g., An et al. 2013).

Recent studies have indicated that a kinematically warmer component associated with the Galactic thick disk could be present in the bulge and would not be part of the bar structure traced out by the majority of the bulge red giants (e.g., Di Matteo et al. 2015). We therefore verified if the bulge RRLs have properties that could link them to the thick disk. However, as seen in Figure 3 (right panel), OGLE-II proper motions (Sumi et al. 2004) of our observed RRLs set them apart from that of the disk, which is not surprising, as it is known that only $\sim 20 \%$ of RRLs in the Milky Way reside in the (thick) disk (Layden 1995). Figure 3 also illustrates how the period distribution of the bulge field RRLs is shifted to longer periods in comparison to the RRL kinematically identified by Layden (1994, 1995) as belonging to the thick disk. Although the local thick disk sample is small (37 stars total), from an RRL pulsational tagging stand point it appears unlikely that the BRAVA-RR component was formed in a similar manner to that of the Milky Way thick disk.

\section{CONCLUSIONS}

It has proven extremely difficult to disentangle the formation history of the inner Galaxy. RRLs are the only luminous evolved stars for which it is possible to place a time stamp: these stars are older than 11 Gyr (Walker 1989). It appears that in the RRL population toward the Galactic bulge, we can observe a distinct stage of the formation of the inner Galaxy that was antecedent to the formation of the bar. This is in agreement with an axisymmetric geometry described using near-infrared VVV observations (Dékány et al. 2013) and is in contrast to the view provided from optical OGLE photometry in which the RRLs appear to follow the elongated spatial distribution of the bar (Pietrukowicz et al. 2015).

The different kinematics of the "bulge" field RRLs and the majority of the bulge giants support the claim that galaxies may harbor two populations in the inner Galaxy, which may be likened to classical and pseudobulges, with differences being with the fraction of the two (Obreja et al. 2013). Within the RRL population toward the direction of the bulge, we can probe an early epoch inner Galaxy that was formed before the massive disk secularly evolved into the bar. Detailed models of the halo, thick disk, and bulge components $\sim 1 \mathrm{kpc}$ from the Galactic center, an understanding of the elemental abundances of the RRLs, as well as a large sample of accurate proper motions for our BRAVA-RR stars, will help distinguish if the "bulge" RRLs reside in a classical-like bulge or are part of a different Milky Way component, such as a metal-rich inner halo-bulge.

We thank the Australian Astronomical Observatory, which have made these observations possible. This research was 
supported in part by the National Science Foundation under grant No. NSF PHY11-25915. This work was supported by Sonderforschungsbereich SFB 881 "The Milky Way System" (subprojects A4, A5, A8) of the German Research Foundation (DFG). R.M.R. acknowledges support from grant AST-1413755 from the National Science Foundation. C.I.J. gratefully acknowledges support from the Clay Fellowship, administered by the Smithsonian Astrophysical Observatory. The research presented here is partially supported by the 973 Program of China under grant No. 2014CB845700, by the National Natural Science Foundation of China under grant Nos.11333003 and 11322326, and by the Strategic Priority Research Program "The Emergence of Cosmological Structures" (No. XDB09000000) of the Chinese Academy of Sciences. J.S. acknowledges support from a Newton Advanced Fellowship awarded by the Royal Society and the Newton Fund. This work made use of the facilities of the Center for High Performance Computing at Shanghai Astronomical Observatory.

\section{REFERENCES}

An, D, Beers, T., Johnson, J. A., et al. 2013, ApJ, 763, 65

Baade, W. 1946, PASP, 58, 249

Babusiaux, C., Gómez, A., Hill, V., et al. 2010, A\&A, 519, 77

Battaglia, G., Helmi, A., Morrison, H., et al. 2006, MNRAS, 370, 1055

Beers, T. C., Chiba, M., Yoshii, Y., et al. 2000, AJ, 119, 2866

Brown, W. R., Geller, M. J., Kenyon, S. J., \& Diaferio, A. 2009, ApJ, 690, 69

Cacciari, C., Clementini, G., \& Buser, R. 1989, A\&A, 209, 154

Cacciari, C., Clementini, G., Prevot, L., et al. 1987, A\&AS, 69, 135

Carney, B. W., \& Latham, D. W. 1984, ApJ, 278, 241

Carretta, E., Bragaglia, A., Gratton, R., D’Orazi, V., \& Lucatello, S. 2009, A\&A, 508, 695

Casey, A. R., \& Schlaufmann, K. C. 2015, ApJ, 809, 110

Chiba, M., \& Beers, T. C. 2000, AJ, 119, 2843

Clementini, G., Cacciari, C., \& Lindgren, H. 1990, A\&AS, 85, 865

Dékány, I., Minniti, D., Catelan, M., et al. 2013, ApJ, 776, 19

Di Matteo, P., Gómez, A., Haywood, M., et al. 2015, A\&A, 577, 1

Fernley, J. A., Skillen, I., Jameson, R. F., et al. 1990, MNRAS, 247, 287

Fiacconi, D., Feldmann, R., \& Mayer, L. 2015, MNRAS, 446, 1957

Fisher, D. B., \& Drory, N. 2011, ApJ, 733, 47

Fisher, D. B., \& Drory, N. 2016, ASSL, 418, 41

Howes, L. M., Casey, A. R., Asplund, M., et al. 2015, Natur, 527, 484

Jeffery, E. J., Barnes, T. G., Skillen, I., \& Montemayor, T. J. 2007, ApJS, 171,512

Johnson, C. I., Rich, R. M., Fulbright, J. P., Valenti, E., \& McWilliam, A. 2011, ApJ, 732, 108

Jones, R. V., Carney, B. W., \& Latham, D. W. 1988, ApJ, 332, 206
Jones, R. V., Carney, B. W., Latham, D. W., \& Kurucz, R. L. 1987, ApJ, 312,254

Kafle, P. R., Sharma, S., Lewis, G. F., \& Bland-Hawthorn, J. 2013, MNRAS, 430, 2973

Koch, A., McWilliam, A., Preston, G. W., \& Thompson, I. B. 2016, A\&A, 587,124

Kormendy, J., \& Illingworth, G. 1982, ApJ, 256, 460

Kovács, G. 2003, MNRAS, 342, 58

Kunder, A., Koch, A., Rich, R. M., et al. 2012, AJ, 143, 57

Kunder, A., Rich, R. M., Hawkins, K., et al. 2015, ApJ, 808, 12

Kunder, A., Stetson, P., Cassisi, S., et al. 2013, AJ, 146, 119

Layden, A. 1994, AJ, 108, 1016

Layden, A. 1995, AJ, 110, 2288

Lee, Y.-W. 1992, AJ, 104, 1780

Lee, Y.-W., Demarque, P., \& Zinn, R. 1994, ApJ, 423, 248

Lee, Y.-W., Joo, S., \& Chung, C. 2015, MNRAS, 453, 3906

Liu, T. 1991, PASP, 103, 205

Liu, T., \& Janes, K. A. 1989, ApJS, 69, 593

Liu, T., \& Janes, K. A. 1990, ApJ, 360, 561

Martig, M., Bournaud, F., Croton, D. J., Dekel, A., \& Teyssier, R. 2012, ApJ, 756, 26

Meylan, G., Burki, G., Rufener, F., et al. 1986, A\&AS, 64, 25

Minchev, I., Famaey, B., Quillen, A. C., et al. 2012, A\&A, 548, 126

Minniti, D. 1994, PASP, 106, 813

Nataf, D. M., Gould, A., Fouqué, P., et al. 2013, ApJ, 769, 88

Ness, M., Freeman, K., Athanassoula, E., et al. 2013, MNRAS, 432, 2092

Noris, J. 1986, ApJS, 61, 667

Obreja, A., Domínguez-Tenreiro, R., Brook, C., et al. 2013, ApJ, 763, 26

Pietrukowicz, P., Udalski, A., Soszyński, I., et al. 2012, ApJ, 750, 169

Pietrukowicz, P., Kozłowski, S., Skowron, J., et al. 2015, ApJ, 811, 113

Renzini, A., \& Buzzoni, A. 1986, ASSL, 122, 195

Rich, R. M. 1990, ApJ, 362, 604

Rich, R. M., Reitzel, D. B., Howard, C. D., \& Zhao, H. 2007, ApJ, 658, 29

Saha, K., \& Gerhard, O. 2013, MNRAS, 430, 2039

Sesar, B. 2012, AJ, 144, 114

Shen, J., Rich, R. M., Kormendy, J., et al. 2010, ApJ, 720, L72

Skillen, I., Fernley, J. A., Stobie, R. S., \& Jameson, R. F. 1993, MNRAS, 265,301

Smolec, R. 2005, AcA, 55, 59

Soszyński, I., Udalski, A., Szyma'nski, M. K., et al. 2014, AcA, 64, 177

Storm, J., Carney, B. W., Latham, D. W., Davis, R. J., \& Laird, J. B. 1992, PASP, 104,168

Storm, J., Nordström, B., Carney, B. W., \& Andersen, J. 1994, A\&A, 291, 121

Sumi, T., Wu, X., Udalski, A., et al. 2004, MNRAS, 348, 1439

Suntzeff, N. B., Kinman, T. D., \& Kraft, R. P. 1991, ApJ, 367, 528

Tumlinson, J. 2010, ApJ, 708, 1398

Walker, A. R. 1989, PASP, 101, 570

Walker, A. R., \& Terndrup, D. M. 1991, ApJ, 378, 119

Wallerstein, G., Gomez, T., \& Huang, W. 2012, Ap\&SS, 341, 89

Zoccali, M., Hill, V., Lecureur, A., et al. 2008, A\&A, 486, 177

Zoccali, M., Gonzalez, O. A., Vasquez, S., et al. 2014, A\&A, 562, 66 